\title{
Pursuit Algorithm for Robot Trash Can Based on Fuzzy-Cell Decomposition
}

\author{
Tatiya Padang Tunggal ${ }^{1}$, Andi Supriyanto ${ }^{2}$, Nur Mukhammad Zaidatur $\mathbf{R}^{3}$, Ibnu Faishal ${ }^{4}$, \\ Imam Pambudi ${ }^{5}$, Iswanto ${ }^{6,7}$ \\ ${ }^{1,7}$ Department of Electro-Medical Engineering Polytechnic, Universitas Muhammadiyah Yogyakarta, Indonesia \\ ${ }^{2,3,4,5,6}$ Department of Electrical Engineering, Universitas Muhammadiyah Yogyakarta, Yogyakarta, Indonesia
}

\begin{tabular}{|c|c|}
\hline Article Info & ABSTRACT \\
\hline Article history: & Scooby Smart Trash can is a trash can equipped with artificial intelligence \\
\hline Received Apr 25, 2016 & $\begin{array}{l}\text { s that is able to capture and clean up garbages thrown by people } \\
\text { ot care about the environment. The can is called smart because }\end{array}$ \\
\hline Revised Oct 28, 2016 & acts like scoobydoo in a children's cartoon in that the can will react if there is \\
\hline Accepted Sep 17, 2016 & garbage thrown and it catches and cleans them up. This paper presents \\
\hline Keyword: & $\begin{array}{l}\text { are used to create a map of the robot's path and fuzzy algorithm as one of the } \\
\text { artificial intelligence algorithm for robot path planning. By using the }\end{array}$ \\
\hline Cell decomposition & combined algorithms, the robot is able to pursuit and chases the trash \\
\hline Fuzzy algorithm & carelessly discarded, but it has not been able to find the shortest distance. \\
\hline Potential field & Therefore, this paper considers a second modification of the algorithm by \\
\hline Pursuit algorithm & adding a potential field algorithm used to add weight values on the map, so \\
\hline Smart trash can & $\begin{array}{l}\text { that the robot can pursue trash by finding the shortest path. The proposed } \\
\text { algorithm shows that the robot can avoid obstacles and find the shortest path } \\
\text { so that the time required to get to the destination point is fast. }\end{array}$ \\
\hline
\end{tabular}

Copyright $(2016$ Institute of Advanced Engineering and Science. All rights reserved.

\section{Corresponding Author:}

Iswanto,

Departement of Electrical Engineering,

Universitas Muhammadiyah Yogyakarta,

Yogyakarta, Indonesia.

Email: iswanto_te@umy.ac.id

\section{INTRODUCTION}

Humans are living beings who want everything looks clean and beautiful including a healthy environment. Many people are concerned about the cleanliness of the surrounding environment but many others are not. It is shown that there are much garbage cluttering around houses, streets and in the city parks. The situation is certainly troubling themselves and users of public facilities.

Having various reasons, many of us are lazy to dispose of trash by walking toward the trash can located. Throwing rubbishes in the bin then becomes one of the ways to dispose them. Sometimes it works, but frequently the garbage thrown on do not go into the trash can and it makes the environment look messy and dirty.

Therefore, some previous researchers have developed a smart robot to dispose trash as practiced by Koyachi et al., [1] developing a robot litter box shaped Hexapoda which is controlled remotely by a camera sensor and an arm that is used to take out the trash. The other researcher named Simoes et al., [2] has developed a medical robot to take out the trash. This wheeled robot is equipped with a camera and a robot arm used to retrieve medical garbage. While Nishida et al., [3] developed a sensor used for robot trash can. Sensors such as camera and laser rangefinder are used to detect trash and the robot autonomously moves toward and takes the trash out and then throw them in the can that has been provided. 
In addition some other researchers have developed robots trash can such as Yamaji et al [4] who developed a robot as a trash can equipped with sensors such as proximity, pyroelectric infrared, infrared and camera sensors. Proximity, pyroelectric infrared, and camera sensors are used to detect people who throw garbage. While the infrared sensor is used to detect the trash that goes into the can. Yang et al. [5] has conducted a research on robot trash can equipped with cameras that enables robot to move autonomously indoors.

The other researcher such as Chiang [6] has embedded a vision camera on the robot trash can used for navigation using two algorithms namely navigation algorithm using genetic algorithms with Simple-Rule and path planner algorithm using Boustrophedon algorithm. Kulkarni \& Junghare [7] have developed a trash detection algorithm by using two ultrasonic sensors embedded on the robot. By using servo motors, the robot can detect tash in a distance range of 2.5 meters with 90 degree angle.

One of the algorithms used by robots to pursue trash which has been investigated by several researchers is cell decomposition algorithm implemented by the diagram Foderaro et al. [8] for pac-man game. The algorithm used to split the map in the game Pac-Man into the grid. The pursuit algorithm was applied to the ghost to chase pac-man.

Cell decomposition algorithm was first investigated by Zhu \& Latombe [9] used for new heuristic path planning algorithm. Cell decomposition algorithm divides the map into similar size grids. The map is configured using c-obstacle algorithm to create three-dimensional obstacles before it is devided into grids. Once a map is created, Search of a Connectivity Graph is used to connect the map paths that have been created with cell decomposition from the starting point to the destination point. This algorithm merely seeks and finds the path, but does not find the shortest path.

The other researchers such as Janchiv et al [10] has examined cell decomposition algorithm used to divide the map into several cells as coverage search areas. In addition, coverage algorithm was used to search in areas that have been mapped. By combining the two algorithms, robots can avoid obstacles and conduct a search in the area.

Path planning algorithms such as $A^{*}$ algorithm combined with a cell decomposition algorithm that is used by Nascimento et al [11] for multiple mobile robots. Cell decomposition algorithm is used to create a map of the neighborhood and the $\mathrm{A}^{*}$ algorithm used to track path so that the robot can avoid obstacles and move towards the goal. In addition, Qureshi and Ayaz [12] have optimized cell decomposition algorithm by combining it with potential field algorithm. The cell decomposition algorithm was used to create a map by dividing the environment into similar size grids and the potential field algorithm was used for path planning.

This paper presents cell decomposition algorithm combined with fuzzy logic algorithm applied to robot trash can. With these algorithms, robot trash can is able to catch and pick the trash carelessly discarded. Additionally robot is also able to avoid obstacles in the environment if there are any in the form of a table or chair.

\section{PURSUIT ALGORITHM}

Pursuit algorithm is an algorithm that searched and calculated the curvature path with used missiles to be able to pursut the target from the initial position toward the end position [13]. Parameters is used by lookahead distance will always get the appropriate path and keep the path to the final position of the aircraft. Besides applied to missile control, the algorithm is applied to the mobile robot is done by Dias et al [14]. Besides apply in the aircraft, the algorithm is examined by Massioni et al., [15] used in pursuing agent for formation.

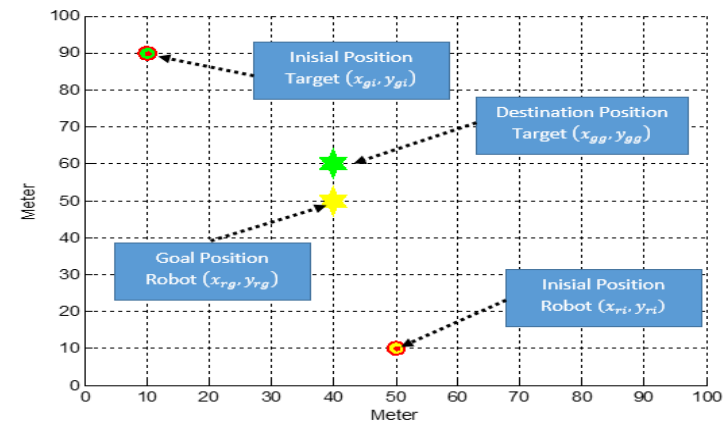

Figure 1. Global view Sensor for pursuit algorithm

IJECE Vol. 6, No. 6, December $2016: 2863-2869$ 
This paper presents pursuit algorithm which has been previously mentioned by the researchers to be applied to the trash can robots that is able to pursue and pick up garbage that has fallen on floor. By using a global view of the sensor, the robot detects the initial position of the trash when discarded and detect the end position of it as illustrated in Figure 1 [16]. It shows that to pursue the target, the robot must have initial position data and end position data of the target so that the end position of the target is the goal of the robot.

\section{MAPPING}

Graph theory have been used by some researchers to create a map of an unknown environment as conducted by Iswanto et al., [16] using cell decomposition methods to create a map of unknown environment by dividing it into several grids and make a robot path planning. Approximate is a part of the cell decomposition method used by Katevas et al., [17] to divide an environment into same grids used for the large wheelchair path planning. A path has been used so that wheelchairs can move to destination position and avoid obstacles.

In this paper, cell decomposition is used to create maps of unknown environments with global view method. With the method the starting position and goal position of the robot, the initial position and final position of the trash and the position of obstacles can be seen as illustrated in Figure 2 [16]. It shows that the starting position of the robot is $\left(x_{r i}, y_{r i}\right)$, and goal position of the robot is $\left(x_{r g}, y_{r g}\right)$, the initial position of the trash is $\left(x_{g i}, y_{g i}\right)$, and final position of the trash is $\left(x_{g g}, y_{g g}\right)$, and the position of obstacles is $\left(x_{o}, y_{o}\right)$. By using the data an environment map is created and divided into grids. The creation and division of the map is carried out by using source code as shown in figure 3 [18].

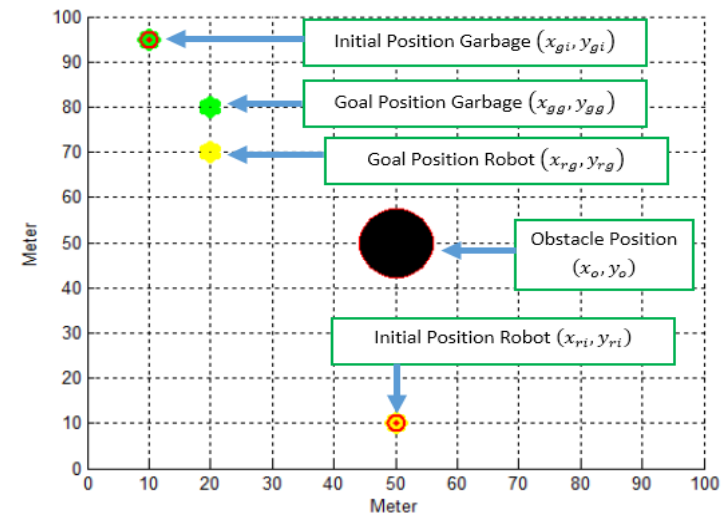

Figure 2. Environment Models

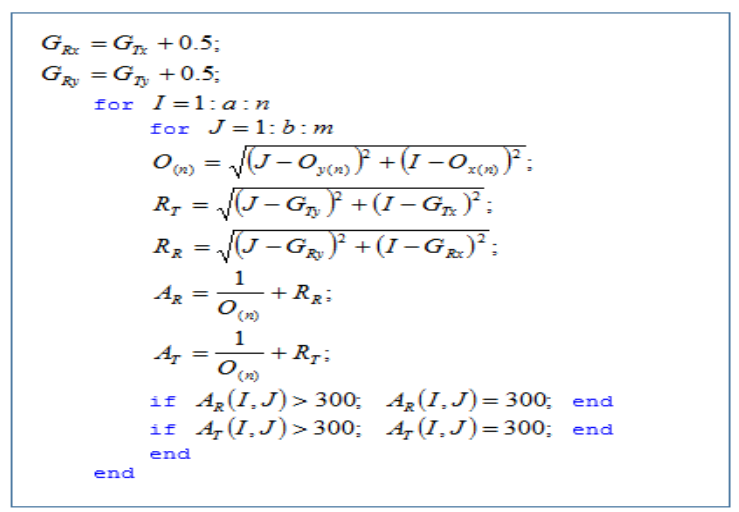

Figure 3. Environment Cell Matrix Decomposition

Figure 3 shows that $\left(G_{R x}, G_{R y}\right)$ is the destination position of the robot, $\left(O_{x(n)}, O_{y(n)}\right)$ is the obstacle's $\mathrm{n}^{\text {th }}$ position, and $\left(G_{T x}, G_{T y}\right)$ is a destination position of the robot. $R_{R}$ is the formula for the distance between the robot position and the destination position and $O_{(n)}$ is the formula of the distance between the robot position and the $\mathrm{n}^{\text {th }}$ obstacles. Then $R_{R}$ and $O_{(n)}$ are summed to obtain a matrix environment value.

\section{FUZZY LOGIC CONTROL ALGORITHM}

Basic behavior in target pursuit in cell decomposition algorithm is that the robot searches the smallest grid and then moves following the grids to reach the destination position. In this study, the trash can robot is controlled by reference values of grid distance obtained from fuzzy algorithm. Fuzzy is an artificial intelligence algorithm [19-22] found by Zadeh that has been used by several researchers to control quadrotor as practiced by Nia Maharani et al [23-24] to control the quadrotor altitude and Mohammad Reza Rahimi et al [25] to control the altitude on the fixed wing UAV type. This algorithm has been widely studied by previous researchers as conducted by Wen et al., [26] using fuzzy algorithm for UAV path planning to fly in dangerous environments at low altitude. In addition, Ge et al., [27] has conducted a research on fuzzy control path planning parameter optimation to be applied to lunar robot which has 6 wheels.

The algorithm applied in this paper for pursuit of targets uses 8 inputs and an output as shown in Figure 4 [18]. It is seen that 8 inputs consist of angles of $0,45,90,135,180,225,270$, and 315 , while the output consists of a corner. 


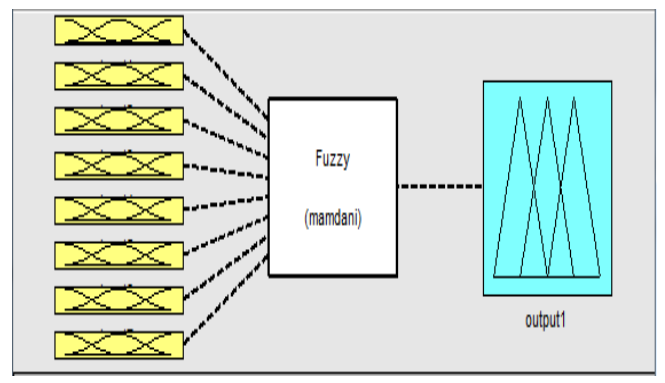

Figure 4. Target Pursuit Design with Fuzzy

The inputs and output of fuzzy sets have different universal set values as shown in Figure 5 [18]. It is seen that the input of fuzzy set has universal values ranging 0-300, while the output of fuzzy set has universal values ranging [-175 175]. There are three members the input set namely Small, medium and big and eight members of the output namely front, front right oblique, left, rear left oblique, rear, rear right oblique, right, and front left oblique.

In fuzzy algorithm, Rule base greatly affects the running proses of a path planning system. This is due to the rule base has a collection of knowledge for decision making in a path planning system shown in Figure 6 [18]. In the picture this basic rule is compared to all the inputs from the global view sensors and the smallest value is determined from the input values to obtain the shortest path.
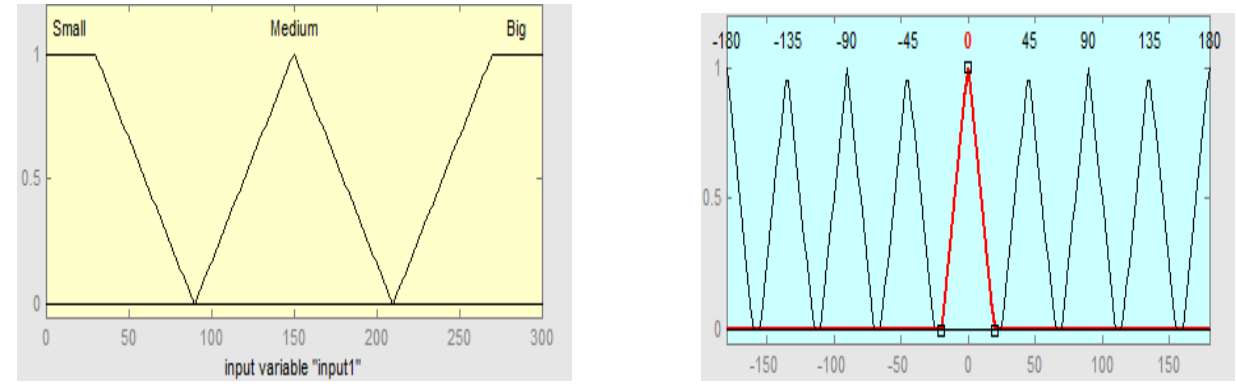

Figure 5. Input and Output Sets of Fuzzy

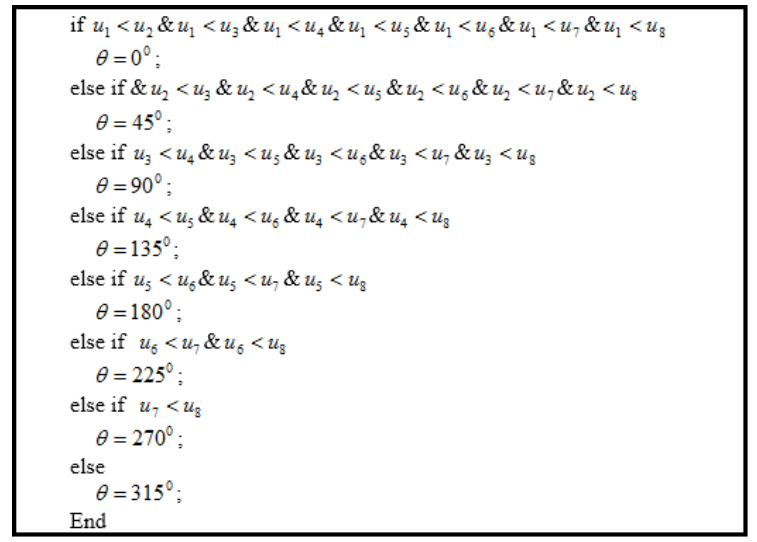

Figure 6. Basic Rules of Path Planning Fuzzy

\section{MODIFIED CELL DECOMPOSITION}

Cell decomposition algorithm has been studied and applied for basic behavior of quadrotorpath planning by Iswanto [16]. By using this algorithm, the robot heads to the goal position by identifying the value of the smallest grid and it avoids obstacles as it get great grid values. The algorithm has been modified 
by adding the value of potential on the grid cell decomposition as shown in Figure 7 [18]. It shows that $k_{O(n)}$ is repulsive force constant for $\mathrm{n}^{\text {th }}$ obstacle and $k_{G R}$ is attractive force constant for the goal position of robot trash can.

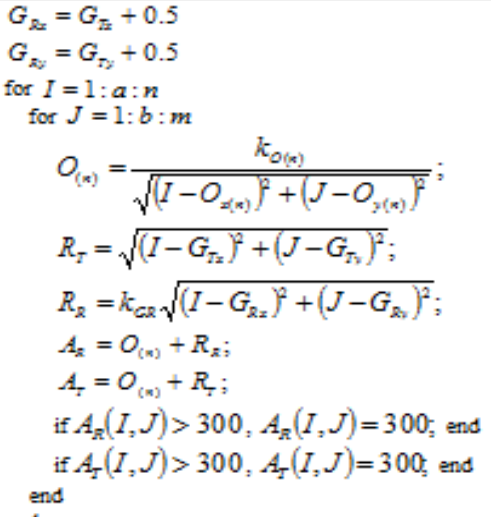

Figure 7. Environtment Matrix Formed by Source Code

\section{RESULT AND ANALYSIS}

The experiments conducted in this paper used matlab software to model the trash, environment and robot and simulated the performance of the robot in pursuing and picking up the trash and avoiding static obstacles in an unknown environment model. There were two experiments in this paper to test the proposed algorithms; the first one was without static obstacles and the second was with static obstacles.
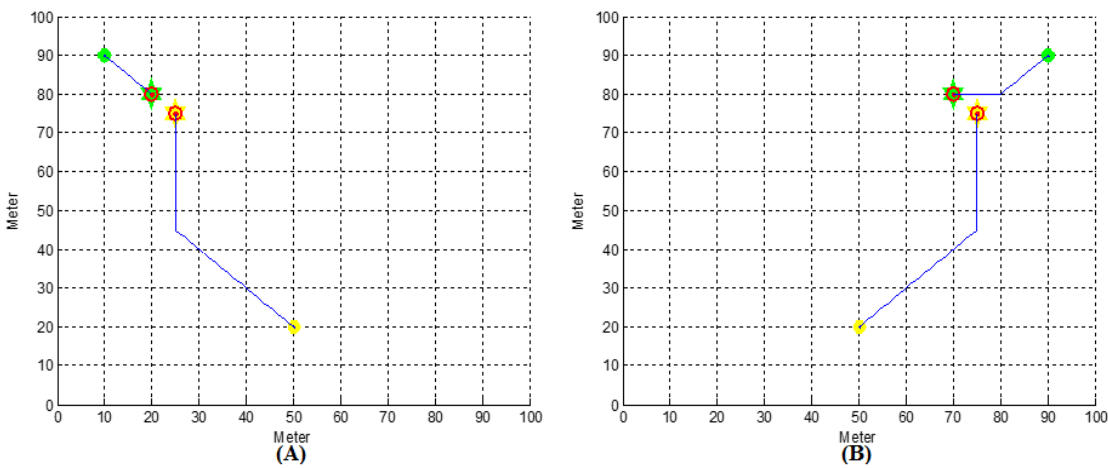

Figure 8. Experiment without Obstacles

The FCD algorithm was tested in the first experiment in an unknown environment without static obstacles as shown in Figure 8. In Figure 8(A) trash can robots is indicated by a yellow dot with the initial position of $(50,20)$ and the robot starts moving when viewed trash indicated a by green dot thrown from the position of $(10,90)$ to the position of $(20,80)$. Then the robot moves toward the goal position of the trash and stop at the position of $(25,75)$. Figure 8 (B) shows that the trash can robot remains at the position of $(50,20)$ and trash indicated by a green dot is in the position of $(90,80)$. When the trash fell on the position of $(80,80)$ the robot moves toward the position of the trash. However, the robot moves back because of the wind and stops at the position of $(70,80)$, and then continues moving towards the position of $(75,75)$.

The second experiment, the robot indicated by a yellow dot was placed at the starting position of $(50,20)$ and there were four obstacles indicated by a black dot placed at four positions of $(30,30),(50,40)$, $(30,60)$, and $(70,60)$ as shown in Figure 9. Figure 9(A) shows that the trash indicated by a green color in the starting position of $(40,90)$ is thrown into the position of $(40,80)$ then the robot pursue it. When heading to the position, the robot encounters some obstacles so it avoids them and continues moving to the nearest position of the trash that is $(45,75)$. Figure 9 (B) shows that the robot trash can is identifying the nearest path

Pursuit Algorithm for Robot Trash Can Based on Fuzzy-Cell Decomposition (Tatiya Padang Tunggal) 
to catch trash and stops at the position $(45,75)$. By using the modified FCD algorithm, the robot pursue the garbage littered more quickly compared to Figure 9 (A).

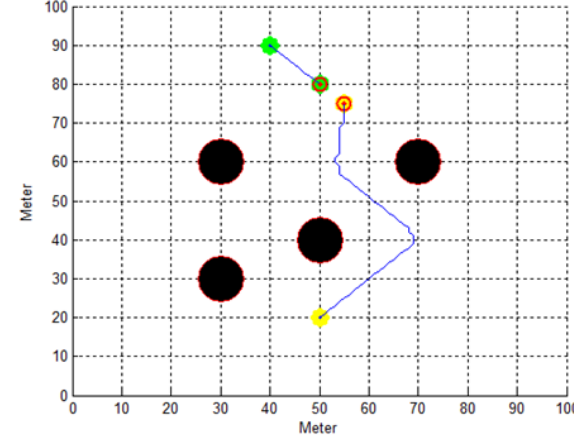

Meter

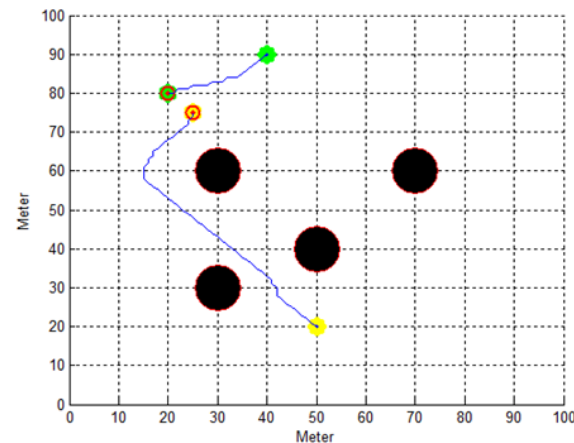

Meter

Figure 9. Experiment using Static Obstacles

\section{CONCLUSION}

The paper presents a new algorithm used by to pursue littered trash based on the combination of cell decomposition algorithm and fuzzy. Fuzzy algorithm is typically used for path planning which serves to detect an obstacle by using sensors and to steer robots towards the goal position. However, in this study the algorithm has been used to detect trash and obstacles read from global view sensors by using cell decomposition algorithm. By combining the two algorithms trash can robots move towards carelessly discarded trash and avoid obstacles.

\section{REFERENCES}

[1] N. Koyachi, H. Adachi, M. Izumi, and T. Hirose, "Control of walk and manipulation by a hexapod with integrated limb mechanism: MELMANTIS-1", in Robotics and Automation, 2002. Proceedings. ICRA '02. IEEE International Conference on, 2002, pp. 3553-3558.

[2] A.S. Simoes, R. Carrion, A.C.G. Martins, R.B. Costa, A.F. Schvarcz, V.R. Buzo, M.H. Felipe, and B.A. Ferrari, "Autonomous mobile robots designing for the medical trash collector task", in Robotics Symposium, 2006. LARS '06. IEEE 3rd Latin American, 2006, pp. 234-239.

[3] T. Nishida, Y. Takemura, Y. Fuchikawa, S. Kurogi, S. Ito, M. Obata, N. Hiratsuka, H. Miyagawa, Y. Watanabe, T. Suehiro, Y. Kawamura, and F. Ohkawa, "Development of a sensor system for outdoor service robot", in SICEICASE, 2006. International Joint Conference, 2006, pp. 2687-2691.

[4] Y. Yamaji, T. Miyake, Y. Yoshiike, P.R. De Silva, and M. Okada, "STB: human-dependent sociable trash box", in Human-Robot Interaction (HRI), 2010 5th ACM/IEEE International Conference on, 2010, pp. 197-198.

[5] S. Yang, B.K.J. Mok, D. Sirkin, H.P. Ive, R. Maheshwari, K. Fischer, and W. Ju, "Experiences Developing Socially Acceptable Interactions for a Robotic Trash Barrel", in Robot and Human Interactive Communication (RO-MAN), 2015 24th IEEE International Symposium on, 2015, pp. 277-284.

[6] C.H. Chiang, "Vision-Based Coverage Navigation for Robot Trash Collection Task," in Advanced Robotics and Intelligent Systems (ARIS), 2015 International Conference on, 2015, pp. 1 - 6.

[7] S. Kulkarni and S. Junghare, "Robot Based Indoor Autonomous Trash Detection Algorithm using Ultrasonic Sensors", in Control, Automation, Robotics and Embedded Systems (CARE), 2013 International Conference on, 2013, pp. $1-5$.

[8] G. Foderaro, V. Raju, and S. Ferrari, "A cell decomposition approach to online evasive path planning and the video game Ms. Pac-Man”, in 2011 IEEE International Symposium on Intelligent Control (ISIC), 2011, pp. 191-197.

[9] D.J. Zhu and J.C. Latombe, "New heuristic algorithms for efficient hierarchical path planning", IEEE Trans. Robot. Autom., vol. 7, no. 1, pp. 9-20, 1991.

[10] A. Janchiv, D. Batsaikhan, B. Kim, W.G. Lee, and S.G. Lee, "Time-efficient and complete coverage path planning based on flow networks for multi-robots", Int. J. Control. Autom. Syst., vol. 11, no. 2, pp. 369-376, 2013.

[11] T.P. do Nascimento, P. Costa, P.G. Costa, A.P. Moreira, and A.G.S. Concei????o, "A set of novel modifications to improve algorithms from the A* family applied in mobile robotics", J. Brazilian Comput. Soc., vol. 19, no. 2, pp. $167-179,2013$.

[12] A. H. Qureshi and Y. Ayaz, "Potential functions based sampling heuristic for optimal path planning", Auton. Robots, vol. 1, pp. 1-3, Nov. 2015.

[13] L. Scharf, W. Harthill, and P. Moose, "A comparison of expected flight times for intercept and pure pursuit missiles”, IEEE Trans. Aerosp. Electron. Syst., vol. AES-5, no. 4, pp. 672-673, Jul. 1969. 
[14] J. Dias, C. Paredes, I. Fonseca, H. Araujo, J. Batista, and A. T. Almeida, "Simulating pursuit with machine experiments with robots and artificial vision", IEEE Trans. Robot. Autom., vol. 14, no. 1, pp. 1-18, 1998.

[15] P. Massioni, F. Ankersen, and M. Verhaegen, "A Matching Pursuit Algorithm Approach to Chaser-Target Formation Flying Problems”, IEEE Trans. Control Syst. Technol., vol. 20, no. 2, pp. 513-519, Mar. 2012.

[16] I. Iswanto, O. Wahyunggoro, and A. Imam Cahyadi, "Path Planning Based on Fuzzy Decision Trees and Potential Field", Int. J. Electr. Comput. Eng., vol. 6, no. 1, p. 212, 2016.

[17] N.I. Katevas, S.G. Tzafestas, and C.G. Pnevmatikatos, "The Approximate Cell Decomposition with Local Node Refinement Global Path Planning Method: Path Nodes Refinement and Curve Parametric Interpolation", J. Intell. Robot. Syst., vol. 22, no. 3-4, pp. 289-314, 1998.

[18] I. Iswanto, O. Wahyunggoro, and A.I. Cahyadi, "Quadrotor Path Planning Based On Modified Fuzzy Cell Decomposition Algorithm”, TELKOMNIKA, vol. 14, no. 2, pp. 655-664, 2016.

[19] T.P. Tunggal, A. Latif, and Iswanto, "Low-cost portable heart rate monitoring based on photoplethysmography and decision tree", in ADVANCES OF SCIENCE AND TECHNOLOGY FOR SOCIETY: Proceedings of the 1st International Conference on Science and Technology 2015 (ICST-2015), 2016, p. 090004.

[20] R. Mubarok, D.V. Firmansyah, D. Haryanto, and N.P. Apriyanto, "Motorcycle-Security using Position Searching Algorithm Based on Hybrid Fuzzy-Dijkstra", Indones. J. Electr. Eng. Comput. Sci., vol. 3, no. 2, pp. 468-474, 2016.

[21] A.N.N. Chamim, D. Ahmadi, and Iswanto, "Atmega16 Implementation As Indicators Of Maximum Speed", Int. J. Appl. Eng. Res., vol. 11, no. 15, pp. 8432-8435, 2016.

[22] I. Iswanto, O. Wahyunggoro, and A.I. Cahyadi, "Hover Position of Quadrotor Based on PD-like Fuzzy Linear Programming", Int. J. Electr. Comput. Eng., vol. 6, no. 5, pp. 2251-2261, 2016.

[23] N.M. Raharja, Iswanto, M. Faris, and A.I. Cahyadi, "Hover position quadrotor control with fuzzy logic", in 2014 The 1st International Conference on Information Technology, Computer, and Electrical Engineering, 2014, pp. 8992.

[24] N.M. Raharja, Iswanto, O. Wahyunggoro, and A.I. Cahyadi, "Altitude control for quadrotor with mamdani fuzzy model”, in 2015 International Conference on Science in Information Technology (ICSITech), 2015, pp. 309-314.

[25] M. Reza, R. Khoygani, S. Hajighasemi, and D. Sanaei, "Designing and Simulation for Vertical Moving Control of UAV System using PID, LQR and Fuzzy logic", Int. J. Electr. Comput. Eng., vol. 3, no. x, p. 8708, 2013.

[26] N. Wen, L. Zhao, X. Su, and P. Ma, "UAV online path planning algorithm in a low altitude dangerous environment", IEEE/CAA J. Autom. Sin., vol. 2, no. 2, pp. 173-185, 2015.

[27] R. Zhang, L. Guo, P. Ge, X. Zhao, and Q. Shao, "Parameter optimisation of path-following fuzzy controller for a six-wheel lunar rover", IET Intell. Transp. Syst., vol. 7, no. 1, pp. 1-9, Mar. 2013. 